\title{
Top-Down Induction of Phylogenetic Trees
}

\author{
Celine Vens ${ }^{1,2}$, Eduardo Costa $^{1}$, Hendrik Blockeel $^{1,3}$ \\ 1 Department of Computer Science, Katholieke Universiteit Leuven, \\ Celestijnenlaan 200A, 3001 Leuven, Belgium \\ 2 Institut National de Recherche Agronomique, UMR 1301, \\ 400 Route des Chappes, 06903 Sophia-Antipolis, France \\ 3 Leiden Institute of Advanced Computer Science, Universiteit Leiden, \\ Niels Bohrweg 1, 2333 CA Leiden, The Netherlands \\ \{celine.vens, eduardo. costa, hendrik.blockeel\}@cs.kuleuven. be
}

\begin{abstract}
We propose a novel distance based method for phylogenetic tree reconstruction. Our method is based on a conceptual clustering method that extends the well-known decision tree learning approach. It starts from a single cluster and repeatedly splits it into subclusters until all sequences form a different cluster. We assume that a split can be described by referring to particular polymorphic locations, which makes such a divisive method computationally feasible. To define the best split, we use a criterion that is close to Neighbor Joining's optimization criterion, namely, minimizing total branch length. A thorough experimental evaluation shows that our method yields phylogenetic trees with an accuracy comparable to that of existing methods. Moreover, it has a number of important advantages. First, by listing the polymorphic locations at the internal nodes, it provides an explanation for the resulting tree topology. Second, the top-down tree growing process can be stopped before a complete tree is generated, yielding an efficient gene or protein subfamily identification approach. Third, the resulting trees can be used as classification trees to classify new sequences into subfamilies.
\end{abstract}

\section{Introduction}

We consider the problem of deriving from a set of aligned DNA or protein sequences the most likely phylogenetic tree. Many methods have been proposed for this. One popular approach consists of computing a dissimilarity measure between each pair of sequences, and then using the resulting matrix to infer the tree. Methods that use this approach are called distance based methods, and are represented by the well-known Neighbor Joining (NJ) [1,2] algorithm.

NJ is essentially a so-called agglomerative hierarchical clustering algorithm: starting from one cluster per sequence, it iteratively merges clusters of sequences until a single cluster is obtained. While agglomerative clustering algorithms are among the most popular ones for clustering, many other clustering algorithms exist. Among these algorithms, one can distinguish extensional and conceptual clustering algorithms. In extensional clustering, a cluster is described by enumerating its elements, whereas in conceptual clustering, a cluster is described 
by listing properties of the cluster's elements, using a particular description language. In the context of phylogenetic analysis, a natural conceptual language would be one that refers to polymorphic locations; for instance, one cluster might be described as "all sequences having a C at position 72 and A at position 31".

If we assume that it must be possible to describe clusters using a particular language, the space of all possible clusterings is greatly reduced. This enables us to use a so-called divisive clustering method, which starts from a single cluster and repeatedly divides it into subclusters until all sequences form a different cluster. Normally, given a set of $N$ sequences, there are $2^{N}$ ways to split it into two subsets. But if we assume that the split can be described by referring to a particular polymorphic location, the number of splits is linear in the length of the sequences, and constant in the size of the set, making such a divisive method computationally feasible, and potentially faster than agglomerative methods. A similar observation was made by [3], who were the first to propose a top-down clustering method for phylogenetic tree reconstruction. Differences between their algorithm and our work are described in the related work section.

Blockeel et al. [4] discuss how a simple extension of decision tree learning leads to a (general-purpose) divisive conceptual clustering algorithm. In this paper we address the question to what extent this approach lends itself to phylogenetic tree reconstruction. If it works well, that is, if it yields phylogenetic trees with an accuracy comparable to that of existing methods, such an approach would have a number of important advantages over existing methods. First, as just argued, it may be faster than methods based on agglomerative clustering. Second, as each division into subclusters is defined by polymorphic locations, the resulting tree immediately gives an evolutionary trace, which can be useful for recognizing functional sites [5]; current methods for this typically involve a two-step process. Third, by using different stopping criteria, the divisive method can be used not only to reconstruct complete phylogenetic trees, but also to identify subfamilies of genes or proteins, without having to grow a complete tree and cutting it afterwards [6]. Finally, since the phylogenetic tree has the form of a classification tree, it can be used directly to classify newly available sequences into such subfamilies, by simply sorting them down the tree into the leaf nodes.

In order to study the top-down conceptual clustering approach in the context of phylogenetic tree reconstruction, we propose a method that is strongly based on an existing decision tree learner; the only change made to it is the heuristic used for splitting nodes. We thoroughly evaluate this new method with respect to accuracy and efficiency. The conclusion is that the method achieves comparable accuracy as existing state-of-the-art methods, with a tendency to perform better for highly symmetric trees, and somewhat worse for highly asymmetric trees.

\section{Background and Related Work}

The Neighbor Joining (NJ) algorithm [1] is one of the most widely used methods for phylogenetic tree reconstruction. It is a heuristic estimation of the minimum evolution tree, which is the tree with minimal sum of branch lengths, and assumes 
a distance matrix with pairwise distances between all sequences is given. It uses a hierarchical clustering approach, at each stage of the clustering grouping two OTUs (Operational Taxonomic Units) together. The original Neighbor Joining algorithm has a complexity $O\left(N^{5}\right)$ [7]. However, Studier and Keppler [2] have presented an alternative version, which is $O\left(N^{3}\right)$. This makes NJ one of the most efficient algorithms for phylogenetic tree reconstruction [8].

Another efficient method, and among all methods the one that most closely resembles ours, is the PTDC (Phylogeny by Top Down Clustering) algorithm [3]. This algorithm shares with our proposal the idea of recursively partitioning clusters. The main differences are: (1) our approach makes the link to the decision tree learning framework, which allows us to exploit the highly developed state of the art in that area; (2) PTDC's heuristic maximizes the average of all pairwise distances between sequences of different clusters, making it similar to the UPGMA algorithm, from which it inherits some undesirable characteristics such as sensitivity to unequal substitution rates in different lineages [9], whereas our method uses a heuristic that approximates the Neighbor Joining criterion; (3) while PTDC splits clusters based on equality of subsequences, our approach creates splits based on a single, most informative, position; this gives it an efficiency advantage.

\section{Method}

As said, we build on the state of the art in decision tree learning. Decision trees are typically built top-down using a recursive partitioning method. Given a dataset and a set of tests (where a test gives one of several possible outcomes when applied to a single data element), they find the test that optimally partitions the dataset into subsets (for some definition of optimal), and keep repeating this procedure on the subsets until subsets of size one are obtained, or another stopping criterion is fulfilled. The procedure is best known in the context of classification trees [10], but it can equally be applied for conceptual clustering [4], in which case tests are considered better if the similarity within the created subsets is higher.

In the context of phylogenetic tree construction, data elements are sequences. A test can for instance check for the occurrence of a particular nucleotide or amino acid at a particular location; this is the kind of tests we use in our method. More specifically, we allow tests of the form " $p(x)=y$ " or " $p(x) \in Y$ ", where $p(x)$ returns the nucleotide or amino acid at position $x$, and where $y$ is a nucleotide or amino acid, and $Y$ a set of them. The test " $p(5) \in\{A, T\}$ ", for instance, creates two subsets, one containing all sequences with an $A$ or $T$ at position 5 and one containing all other sequences.

Decision tree learners typically stop partitioning sets when all elements of the set are sufficiently similar, e.g., for classification trees, when all elements belong to the same class. For phylogenetic trees, one could do something similar, if the tree need only be built up to the level of subfamilies; here, however, we will focus on building complete trees, i.e., we keep splitting until all subsets are singletons. 


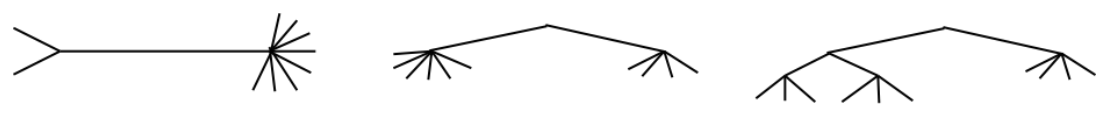

Fig. 1. Split topologies. Left: NJ, center: Clus- $\varphi$ (root), right: Clus- $\varphi$ (non-root).

The only question that remains, then, is how to determine the quality of a split. Intuitively, one could argue that an "old" mutation, i.e., one that occurred a long time ago in evolutionary history, is more likely to have led to two different branches that by now have grown far apart, than a more recent mutation. As we are building a rooted tree, where we hope to find older mutations nearer to the root, we should prefer tests that create subsets that are far apart. A natural heuristic for selecting tests is then simply: compute the average distance between elements from different subsets induced by the test; choose the test that maximizes that average distance. This is the heuristic that Arslan et al. use [3]. Alternatively, one could use an extension of the information gain heuristic that is commonly used for classification trees; Clare et al. [11] define such an extension for multilabel classification trees, and this heuristic is available in the decision tree learner on which our system is based.

These standard heuristics, however, do not take into account the particular requirements of phylogenetic tree reconstruction. Using the average distance heuristic, one essentially gets the top-down counterpart of the UPGMA algorithm, which is known to have some undesirable behavior [8]. NJ works in essentially the same way as UPGMA, but uses a more advanced distance metric for deciding which two clusters to merge, and this yields better results. This raises the question whether a top-down counterpart of NJ's heuristic can be developed.

Such a heuristic would have to estimate the total branch length of the tree that will finally be constructed, using the pairwise distances provided in a distance matrix $D$. Using the same reasoning as in the derivation of NJ's heuristic [1], we can define an equivalent heuristic function for splitting the root node:

$$
H\left(T_{l}, T_{r}\right)=\frac{1}{\left|T_{l}\right|\left|T_{r}\right|} \sum_{\substack{x_{i} \in T_{l} \\ x_{j} \in T_{r}}} D_{x_{i} x_{j}}+\frac{1}{\left|T_{l}\right|} \sum_{\substack{x_{i}, x_{j} \in T_{l} \\ i<j}} D_{x_{i} x_{j}}+\frac{1}{\left|T_{r}\right|} \sum_{\substack{x_{i}, x_{j} \in T_{r} \\ i<j}} D_{x_{i} x_{j}},
$$

where $T_{l}$ and $T_{r}$ denote the set of sequences in the left and right subtrees of the split, respectively. This formula computes the total branch length of a "double star"-shaped topology (see Fig 1, center), which is an upper bound for the actual total branch length of the final tree. It is a generalization of the NJ heuristic, which has $\left|T_{l}\right|=2$ and $\left|T_{r}\right|=N-2$, with $N$ the total number of sequences (Fig 1, left). The previous equation can be rewritten into a more efficient formulation,

$$
\begin{aligned}
H\left(T_{l}, T_{r}\right)= & \frac{1}{\left|T_{l}\right|\left|T_{r}\right|}\left(\sum_{\substack{x_{i}, x_{j} \in T_{l} \cup T_{r} \\
i<j}} D_{x_{i} x_{j}}+\left(\left|T_{r}\right|-1\right) \sum_{\substack{x_{i}, x_{j} \in T_{l} \\
i<j}} D_{x_{i} x_{j}}+\right. \\
& \left.\left(\left|T_{l}\right|-1\right) \sum_{\substack{x_{i}, x_{j} \in T_{r} \\
i<j}} D_{x_{i} x_{j}}\right)
\end{aligned}
$$


where the first term is constant for the node to be split.

For splitting the other internal nodes, a more complex heuristic function is needed, since the particular split influences the length of other branches in the tree, and hence, the total branch length (Fig 1, right). Again, using a similar reasoning as applied by Saitou and Nei [1], it can be verified that the function to minimize is given by

$$
\begin{aligned}
H\left(T_{l}, T_{r}, T_{o}\right)= & \frac{1}{\left|T_{l}\right|\left|T_{r}\right|}\left(\sum_{x_{i}, x_{j} \in T_{i} \cup T_{r}} D_{x_{i} x_{j}}+\left(2\left|T_{r}\right|-1\right) \sum_{\substack{x_{i}, x_{j} \in T_{l} \\
i<j}} D_{x_{i} x_{j}}+\right. \\
& \left.\left(2\left|T_{l}\right|-1\right) \sum_{\substack{x_{i}, x_{j} \in T_{r} \\
i<j}} D_{x_{i} x_{j}}+\frac{\left|T_{r}\right|}{\left|T_{o}\right|} \sum_{\substack{x_{i} \in T_{o} \\
x_{j} \in T_{l}}} D_{x_{i} x_{j}}+\frac{\left|T_{l}\right|}{\left|T_{o}\right|} \sum_{\substack{x_{i} \in T_{o} \\
x_{j} \in T_{r}}} D_{x_{i} x_{j}}\right),(3)
\end{aligned}
$$

with $T_{o}$ denoting the set of other sequences in the tree (i.e., not in $T_{l} \cup T_{r}$ ).

The computational complexity of a decision tree learning method is roughly $O(a N \log N)$ with $a$ the number of tests and $N$ the number of elements in the original dataset, under the assumption that a reasonably symmetric tree is built (the depth of which is logarithmic in the number of leaves) and the evaluation of a single test takes linear time in the size of the dataset. This scales much better in $N$ than agglomerative methods, which have complexity $O\left(N^{3}\right)$ [2]. As such, such a divisive method may be much more efficient when analyzing large sets of sequences. The NJ-based heuristic, however, is more expensive to compute than the average distance or information gain heuristics; it is quadratic, rather than linear, in the size of the dataset being subdivided, which increases the overall complexity of the method to $O\left(a N^{2} \log N\right)$.

Our algorithm is called CLUS- $\varphi$ and is implemented in the Clus system (http://www.cs.kuleuven.be/ ${ }^{\text {dtai/clus). }}$

\section{Experiments}

We have tested our alternative method for phylogenetic tree construction on a number of datasets. In all experiments, we used the Jukes-Cantor correction formula [12] to compute the genetic distance between two DNA sequences, and the Jones-Taylor-Thornton matrix [13] for protein sequences. We measure differences between trees using the quartet distance [14]. This distance gives the number of quartets, i.e. subtrees induced by four leaves, that differ between the two trees being compared.

\subsection{Real datasets}

In a first set of experiments, we check how much CLUS- $\varphi$ trees differ from the ones returned by Neighbor Joining (NJ). As a reference point, we include the difference between parsimony methods and $\mathrm{NJ}^{4}$. To construct the NJ and parsimony trees we used the programs neighbor and dnapars/protpars from the Phylip software package [15], respectively.

\footnotetext{
${ }^{4}$ When parsimony analysis returns multiple trees, we report the average difference.
} 
Table 1. Quartet distance for real datasets.

\begin{tabular}{lccccc}
\hline Dataset & Type & Size & Length & NJ vs. Clus- $\varphi$ & NJ vs. Pars \\
\hline Chimp & DNA & 5 & 896 & 0 & 2 \\
cynmix & DNA & 32 & 3080 & 11680 & 16543 \\
Primates & DNA & 21 & 1500 & 184 & 632 \\
SIV & DNA & 41 & 6042 & 21350 & 10608 \\
hivALN & DNA & 14 & 2352 & 0 & 156 \\
InvertebrateEF & DNA & 16 & 1050 & 200 & 152 \\
mtDNA & DNA & 17 & 1998 & 218 & 26 \\
VertebrateMtCOI & DNA & 8 & 1509 & 178 & 31 \\
g3pdh & Protein & 14 & 313 & 180 & 65.33 \\
gpd & Protein & 12 & 234 & 52 & 18 \\
gdpAA & Protein & 12 & 422 & 73 & 97.50 \\
\hline
\end{tabular}

Table 1 reports the quartet distance for 11 datasets used in [8]. While the trees generated by CLUS- $\varphi$ are very similar to those generated by NJ for some datasets (for datasets Chimp and hivALN, CLUS- $\varphi$ and NJ generated identical phylogenetic trees), there are larger differences for other datasets. However, this variation is comparable to the variation between the parsimony method and NJ.

The question is then whether, in those cases where NJ and Clus- $\varphi$ differ, any of them is more likely to be correct than the other one. To check this, we tested Clus- $\varphi$ on a number of synthetic datasets, where the correct tree is known. These experiments are discussed in the next section.

\subsection{Synthetic datasets}

The synthetic datasets were generated by simulating an evolutionary process, using the coding sequence simulation program EvolveAGene3 [16], with a randomly generated DNA sequence as input. By default, this software produces symmetric trees, i.e., binary trees that have all leaves at the same depth. However, also random tree topologies can be produced. The average branch length is set to 0.05 in all experiments, meaning that each branch has an average mutation rate of $5 \%$. All other parameters are set to their default value.

First, we evaluate our proposed heuristic (see Section 3). Second, we compare the trees obtained by CLUS- $\varphi$ to those obtained by NJ in terms of similarity with the true tree topology. Finally, we investigate the influence of the number of sequences on quartet distance and computational cost for both NJ and CLUS- $\varphi$.

\section{Analysis of the heuristic}

To evaluate our proposed heuristic, we compare trees constructed using our heuristic to trees constructed with two other heuristics for decision trees. The first heuristic is an extension of the standard information gain heuristic towards multilabel classification [11]; we call this implementation CLUS-IG. The second heuristic is similar ${ }^{5}$ to the one used in the PTDC algorithm [3]. It recursively

\footnotetext{
${ }^{5}$ The only difference between our implementation and the one discussed in [3] is that we create splits based on a single position instead of based on subsequences.
} 
Table 2. Number of wins/losses of CLUS- $\varphi$ 's heuristic compared to information gain (Clus-IG) and inter-cluster distance (CLUS-PTDC).

\begin{tabular}{ccc}
\hline & Clus- $\varphi$ vs Clus-IG & Clus- $\varphi$ vs Clus-PTDC \\
\hline Symmetric & $113 / 78(9$ ties $)$ & $200 / 0$ \\
Random & $113 / 87$ & $200 / 0$ \\
\hline
\end{tabular}

splits the data by looking, in each step, for the two subclusters with the largest average inter-cluster distance. We call this implementation CLus-PTDC.

In order to perform this experiment, we generated 400 synthetic datasets: 200 based on symmetric topologies and 200 based on random topologies, with an input sequence length of 250 (200 datasets) and 900 (200 datasets) ${ }^{6}$, each dataset containing 128 sequences. Table 2 presents a summary of the results, in terms of the number of wins/losses of CLUS- $\varphi$ in the comparison with CLUS-IG and Clus-PTDC, according to the quartet distance from the inferred trees to the true tree. As can be seen from the results, CLUS- $\varphi$ presents a higher number of wins than CLus-IG, and is better than CLUS-PTDC for all datasets. It shows that, in the context of phylogenetic tree reconstruction, the new heuristic yields better results than standard heuristics for decision trees.

\section{Comparison between Clus- $\varphi$ and NJ in terms of similarity to the true tree topology}

In this experiment we compare the similarity with the true tree for NJ and CLUS$\varphi$. We generated 100 synthetic datasets based on symmetric topologies and 100 datasets based on random topologies, containing 128 sequences and using an input sequence of length 900 . A summary of the results, in terms of the number of datasets for which each method presents the best performance, is shown in Table 3. On average, NJ performs slightly better than CLUS- $\varphi$; however the table shows a large difference in the results for symmetric and random tree topologies. For symmetric trees, the CLUS- $\varphi$ tree is in general closer to the true tree than the tree produced by NJ. For the random tree topologies, on the other hand, Clus- $\varphi$ is closer to the true tree in only 5 cases. The reason for this difference in behavior is currently unclear.

Interestingly, we noted that in a few cases, the trees produced by Clus- $\varphi$ are identical to the true topologies; this occurred for 5 out of 100 cases for the datasets generated from symmetric topologies. For NJ, that is never the case. The probability of observing at most 0 correct predictions for NJ and at least 5 for CLUS- $\varphi$, under the null hypothesis that both have the same probability $p$ of making a correct prediction, depends on $p$ but is always less than 0.01 ; this implies that the hypothesis that CLus- $\varphi$ does not have a higher probability of predicting the correct tree than NJ is rejected at the $1 \%$ significance level.

\footnotetext{
${ }^{6}$ As insertions and deletions are allowed in EvolveAGene3, the final sequence length is usually larger than the input sequence length, e.g. for an input sequence of length 900 , the length of the final sequences is around 1000.
} 
Table 3. Number of wins of NJ and CLUS- $\varphi$ for synthetic datasets.

\begin{tabular}{ccc}
\hline & NJ & Clus- $\varphi$ \\
\hline Symmetric & 32 & $\mathbf{6 8}$ \\
Random & $\mathbf{9 5}$ & 5 \\
\hline
\end{tabular}
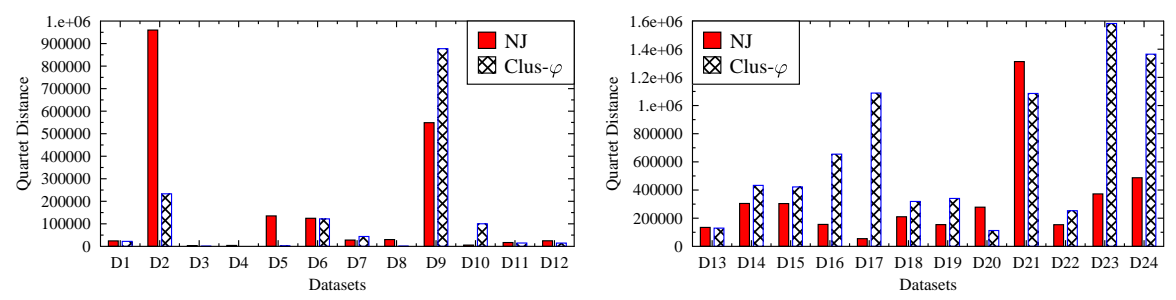

\begin{tabular}{cccccc}
\hline D1 & D2 & D3 & D4 & D5 & D6 \\
\hline 0.91 & 0.24 & 0.16 & 0.00 & 0.01 & 0.98 \\
\hline D7 & D8 & D9 & D10 & D11 & D12 \\
\hline 1.57 & 0.02 & 1.60 & 17.63 & 0.87 & 0.59 \\
\hline
\end{tabular}

\begin{tabular}{cccccc}
\hline D13 & D14 & D15 & D16 & D17 & D18 \\
\hline 0.96 & 1.42 & 1.39 & 4.20 & 19.88 & 1.52 \\
\hline D19 & D20 & D21 & D22 & D23 & D24 \\
\hline 2.20 & 0.40 & 0.83 & 1.64 & 4.25 & 2.80 \\
\hline
\end{tabular}

Fig. 2. Quartet distance to real tree, for symmetric (left) and random (right) trees. Graphs: absolute values; Tables: quartet distance of CLUS- $\varphi$ relative to that of NJ.

In Figure 2 we show the quartet distance for 12 datasets of each kind, in order to analyze the results in more detail. We can see that for random trees the differences between the two methods are larger. The graphs also show the strong variation of tree quality with the dataset: the dataset has a larger influence on the overall performance than the choice of method.

From the results shown in Table 3 and Fig. 2 we can conclude that CLus- $\varphi$ tends to perform better for symmetric tree topologies, while NJ tends to perform better for random topologies. In a sense, these settings are at both ends of a spectrum. The question is then how the results differ for datasets based on trees that are neither perfectly symmetric nor completely random, which is what we expect to occur in nature.

To investigate this question, we have generated datasets based on a series of tree topologies, starting from a perfectly symmetric tree, and gradually adding more random tree structure. More precisely, we considered a tree operation that takes two subtrees, one consisting of a single leaf, and another one consisting of one internal node and two leaves, and switches them. Table 4 reports the results for an experiment that counts the number of wins for NJ and CLUS- $\varphi$ on 100 datasets with an increasing number of operations. Each dataset again has 128 sequences generated from a DNA input sequence of 900 positions.

As can be seen from the results, the performance of CLUS- $\varphi$, compared to NJ, decreases with the increase of the number of modifications in the symmetric tree. However, it is important to notice that this decrease of the performance of Clus- $\varphi$ occurs gradually, which means that it presents good results not only for 
Table 4. Analysis of the effect of the symmetry of the tree on the performance of NJ and CLUS- $\varphi$. The numbers in bold represent the largest number of wins for each line.

\begin{tabular}{ccc}
\hline Dataset & NJ & CLUS- $\varphi$ \\
\hline Symmetric & 32 & $\mathbf{6 8}$ \\
5 steps & $\mathbf{5 6}$ & 44 \\
10 steps & $\mathbf{5 6}$ & 44 \\
15 steps & $\mathbf{5 3}$ & 47 \\
20 steps & $\mathbf{6 8}$ & 32 \\
25 steps & $\mathbf{6 5}$ & 35 \\
30 steps & $\mathbf{6 3}$ & 37 \\
\hline
\end{tabular}

\begin{tabular}{ccc}
\hline Dataset & NJ & CLUS- $\varphi$ \\
\hline 35 steps & $\mathbf{6 5}$ & 35 \\
40 steps & $\mathbf{6 6}$ & 34 \\
45 steps & $\mathbf{7 7}$ & 23 \\
50 steps & $\mathbf{7 6}$ & 24 \\
55 steps & $\mathbf{7 7}$ & 23 \\
60 steps & $\mathbf{8 1}$ & 19 \\
Random & $\mathbf{9 5}$ & 5 \\
\hline
\end{tabular}

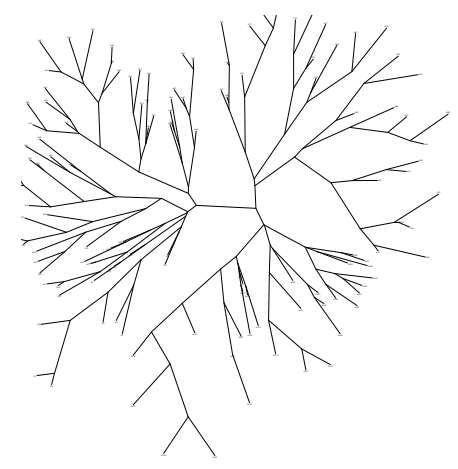

Fig. 3. Tree topology with 40 steps from symmetry.

completely symmetric trees, but also for almost symmetric trees: up till 40 steps, CLUS- $\varphi$ finds a better approximation of the true tree in one out of three datasets. As an illustration, Fig. 3 shows a topology with 40 steps from symmetry.

\section{Analysis of the influence of the number of sequences on quartet distance and computational cost}

In this section, we analyze the effect of the number of sequences on the performance of the algorithms, both in terms of tree reconstruction, and computational cost. For this analysis, we use datasets based on symmetric tree topologies.

To analyze how NJ and CLUS- $\varphi$ scale in the number of sequences, a number of synthetic datasets that contain sequences of the same length ${ }^{7}$, but with an increasing number of sequences were generated.

Figure 4 (left) shows run times for datasets with 300 nucleotides. Each point in the curve shows the average run times over 20 datasets of the specified size. As we can see, between 1000 and 2000 sequences, the run times of CLUS- $\varphi$ become smaller than those of NJ. For 8000 sequences, CLus- $\varphi$ is about 5 times faster (the exact values are 1517 seconds for CLUS- $\varphi$ and 7990 seconds for NJ).

\footnotetext{
$\overline{7}$ To ensure an equal dataset length, we disabled insertions and deletions here.
} 

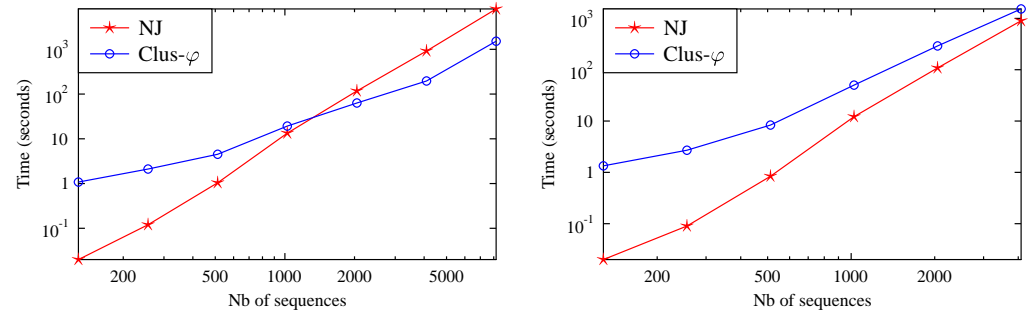

Fig. 4. Running times for CLUS- $\varphi$ and NJ (logarithmic scale). Sequence length: 300 (left) and 900 (right) nucleotides.

Table 5. Number of wins of NJ and CLUS- $\varphi$ for synthetic datasets with sequences of length 900 according to the quartet distance.

\begin{tabular}{cccc}
\hline Nb Sequences & NJ & Clus- $\varphi$ & Ties \\
\hline 8 & 0 & $\mathbf{1}$ & 19 \\
16 & 2 & $\mathbf{7}$ & 11 \\
32 & 2 & $\mathbf{9}$ & 9 \\
64 & 5 & $\mathbf{1 3}$ & 2 \\
128 & 6 & $\mathbf{1 4}$ & 0 \\
256 & $\mathbf{1 1}$ & 9 & 0 \\
512 & $\mathbf{1 3}$ & 7 & 0 \\
1024 & $\mathbf{1 3}$ & 7 & 0 \\
2048 & $\mathbf{1 8}$ & 2 & 0 \\
4096 & $\mathbf{1 5}$ & 5 & 0 \\
\hline
\end{tabular}

Since our method scales linearly in the sequence length (as each split requires inspecting all positions; see Sect. 3), while NJ is constant in the length, we also show the run times for datasets with longer sequences. Figure 4 (right) shows that NJ is more efficient than CLUS- $\varphi$ for datasets with 900 nucleotides. However, as the relative run time difference between both methods decreases, we expect that CLUS- $\varphi$ will be faster than NJ for datasets with more than 4000 sequences $^{8}$.

We also computed the quartet distance of the trees produced by NJ and Clus- $\varphi$ to the true tree for the datasets with 900 nucleotides (see Table 5). It can be noted that CLuS- $\varphi$ obtains better results than NJ for datasets with few sequences. However, between 128 and 256 sequences, the results change, making NJ the best method for a large number of sequences. The reason for this is related to the relatively small sequence length: in order to generate 256 sequences or more with only 900 nucleotides, each branch having on average 45 mutations, a lot of nucleotide positions are likely to have many mutations. This negatively influences CLUS- $\varphi$, because it can not find the right splits anymore. How this can be dealt with (e.g. by post-processing the trees using local rearrangements) will be investigated in future work.

\footnotetext{
${ }^{8}$ It is infeasible to generate datasets with more than 4000 sequences of length 900 with the EvolveAGene3 program.
} 


\subsection{Comparison to PTDC}

As a last experiment, we compare CLus- $\varphi$ to PTDC [3]. The authors have used a single dataset to evaluate their method and report the resulting tree in their article. The dataset was prepared by Rennert et al. [17], who also report a maximum likelihood tree for the alignment, which we use as reference tree. For this experiment, both PTDC and CLUS- $\varphi$ calculate pairwise protein distances using the PAM1 substitution matrix [18]. Given that the reference tree in [17] is not binary (it contains a node with six branches), it is impossible to calculate the quartet distance. Therefore, for this experiment, we compare the trees using the symmetric difference measure. The symmetric difference between two trees is the number of binary node partitions that are found in one tree and not in the other. The symmetric difference to the reference tree is 11 for PTDC, and 10 for Clus- $\varphi$. We conclude that our method finds a slightly better tree.

\section{Conclusion}

We have proposed CLuS- $\varphi$, a novel method for reconstruction of phylogenetic trees. The method differs strongly from state-of-the-art methods, both from an algorithmic point of view and from the point of view of the information it uses.

It is based on a conceptual clustering method that extends the well-known decision tree learning approach. Starting from a single cluster, it repeatedly splits the sequences into subclusters until all sequences form a different cluster, or until a sufficient level of detail is obtained to study gene or protein families. To define the best split, our method uses a criterion that is close to Neighbor Joining's optimization criterion, namely, constructing a phylogenetic tree with minimal total branch length.

Our method assumes that a split can be described by referring to particular polymorphic locations, which makes such a divisive method computationally feasible, and at the same time provides an evolutionary trace for the resulting tree topology. Moreover, by checking the polymorphic locations listed in the internal nodes, new sequences can easily be classified into subfamilies.

We have shown that the performance of CLUS- $\varphi$ is close to that of Neighbor Joining w.r.t. quality of the produced trees, while having a larger tendency to produce the correct tree.

We propose CLUS- $\varphi$ not as a substitute for Neighbor Joining or other standard methods for phylogeny reconstruction, but as a method to be used complementarily to these methods. The fact that CLUS- $\varphi$ behaves differently than Neighbor Joining, in terms of performance and computational cost, shows that their results can be used to complement one another.

\section{Acknowledgments}

Celine Vens is a Postdoctoral Fellow of the Research Foundation - Flanders (FWO). Eduardo Costa is supported by the Research Foundation - Flanders (FWO) and the GOA Probabilistic Logic Learning. The authors would like to thank Etienne Danchin for providing useful suggestions to improve the text. 


\section{References}

1. Saitou, N., Nei., M.: The neighbor-joining method: a new method for reconstructing phylogenetic trees. Mol. Biol. Evol. 4(4) (1987)

2. Studier, J., Keppler, K.: A note on the Neighbor-Joining algorithm of Saitou and Nei. Mol. Biol. Evol. 5(6) (1988)

3. Arslan, A.N., Bizargity, P.: Phylogeny by top down clustering using a given multiple alignment. In: Proceedings of the 7th IEEE Symposium on Bioinformatics and Biotechnology (BIBE 2007), Vol. II. (2007)

4. Blockeel, H., De Raedt, L., Ramon, J.: Top-down induction of clustering trees. In: Proceedings of the 15th International Conference on Machine Learning. (1998)

5. Morgan, D., Kristensen, D., Mittelman, D., Lichtarge, O.: ET Viewer: an application for predicting and visualizing functional sites in protein structures. Bioinformatics 22(16) (2006)

6. Brown, D.P., Krishnamurthy, N., Sjlander, K.: Automated protein subfamily identification and classification. PLoS Computational Biology 3 (2007)

7. Gascuel, O.: A note on Sattath and Tversky's, Saitou and Nei's, and Studier and Keppler's algorithms for inferring phylogenies from evolutionary distances. Mol. Biol. Evol. 11(6) (1994)

8. Salemi, M., Vandamme, A.: The Phylogenetic Handbook: A Practical Approach to DNA and Protein Phylogeny. Cambridge University Press (2003)

9. Sourdis, J., Krimbas, C.: Accuracy of phylogenetic trees estimated from DNA sequence data. Molecular Biology and Evolution 4 (1987)

10. Quinlan, J.: C4.5: Programs for Machine Learning. Morgan Kaufmann series in Machine Learning. Morgan Kaufmann (1993)

11. Clare, A., Karwath, A., Ougham, H., King, R.D.: Functional bioinformatics for Arabidopsis thaliana. Bioinformatics 22(9) (2006)

12. Jukes, T., Cantor, C.: Evolution of protein molecules. In Munro, H., ed.: Mammalian Protein Metabolism. Academic Press, New York (1969)

13. Jones, D., Taylor, W., Thornton, J.: The rapid generation of mutation data matrices from protein sequences. Computer Applications in the Biosciences 8 (1992)

14. Estabrook, G., McMorris, F., Meacham, C.: Comparison of undirected phylogenetic trees based on subtrees of four evolutionary units. Systematic Zoology 34 (1985)

15. Felsenstein, J.: Phylip (phylogeny inference package) version 3.68 (2008) http://evolution.genetics.washington.edu/phylip.html.

16. Hall, B.: Simulating DNA Coding Sequence Evolution with EvolveAGene3. Molecular Biology and Evolution 25(4) (2008)

17. Rennert, J., Coffman, J., Mushegian, A., Robertson, A.: The evolution of Runx genes I. A comparative study of sequences from phylogenetically diverse model organisms. BMC Evolutionary Biology 3(4) (2003)

18. Dayhoff, M., Schwartz, R., Orcutt, B.: A model of evolutionary change in proteins. Atlas of protein sequence and structure 5(suppl. 3) (1978) 